\title{
Trastuzumab deruxtecan for the treatment of HER2-positive advanced gastric cancer: a clinical perspective
}

\author{
Masahiko Aoki ${ }^{1} \cdot$ Satoru Iwasa ${ }^{1} \cdot$ Narikazu Boku $^{1}$ (D) \\ Received: 2 December 2020 / Accepted: 22 January 2021 / Published online: 1 March 2021 \\ (c) The International Gastric Cancer Association and The Japanese Gastric Cancer Association 2021
}

\begin{abstract}
Human epidermal growth factor receptor 2 (HER2)-positive gastric cancer is a subtype for which new drugs and specific treatment strategies should be developed. Trastuzumab deruxtecan (T-DXd) is a novel HER2-targeted antibody-drug conjugate containing topoisomerase I inhibitor as a payload. In the randomized phase 2 study (DESTINY-Gastric01) for HER2-positive advanced gastric or gastroesophageal junction cancer (AGC), patients treated with T-DXd showed a significantly higher response rate compared with the chemotherapy of physician's choice, associated with remarkably prolonged progressionfree and overall survival. T-DXd also exhibits anti-tumor activity to HER2-negative tumor cells close to HER2-positive cells (so-called bystander killing effect). T-DXd was effective even for HER2-low expressing breast and gastric cancer in several clinical studies. Taking advantage of these strong points and synergism with other cytotoxic, molecular-targeted and immunological agents, it is expected that T-DXd will bring further progression in treatment both for strongly and weakly HER2 positive AGC in various treatment settings including perioperative chemotherapy.
\end{abstract}

Keywords Human epidermal growth factor receptor $2($ HER2) $\cdot$ Gastric cancer $\cdot$ Trastuzumab deruxtecan (T-DXd)

\section{Introduction}

Human epidermal growth factor receptor 2 (HER2) is a $185 \mathrm{kDa}$ protein with tyrosine kinase activity that belongs to the epidermal growth factor receptor (EGFR) family. HER2 is associated with increased tumor cell proliferation, reduced apoptosis, and enhanced migration. Indeed, HER2positive breast cancers have a significantly poorer prognosis than HER2-negative breast cancers if patients are not placed on anti-HER2 therapy [1]. Clinical trials of trastuzumab (a monoclonal antibody that binds to the extracellular domain of HER2) began in 1992, and its efficacy in HER2-positive breast cancer led to its first approval in 1998 [2].

Gastric cancer (GC) is the third-leading cause of cancerrelated deaths worldwide [3]. Even though various chemotherapeutic agents have been developed for advanced gastric/ gastroesophageal junction cancer (AGC), median overall survival (OS) of AGC patients is about 1 year. Amplification

Narikazu Boku

nboku@ncc.go.jp

1 Division of Gastrointestinal Medical Oncology, National Cancer Center Hospital, 5-1-1, Tsukiji, Chuo-ku,

Tokyo 104-0045, Japan of HER2 gene or overexpression of HER2 protein are found in 10-20\% of GC [4]; American Society of Clinical Oncology/College of American Pathologists guidelines define HER2-positive GC as immunohistochemistry (IHC) $3+$, or IHC $2+$ and in situ hybridization (ISH) + [5]. While HER2positive AGC is reported to be aggressive [4], the prognostic impact of HER2-status is still controversial [6, 7].

Several clinical studies for HER2-positive AGC have been conducted (Table 1). In the ToGA study [8], patients received chemotherapy or chemotherapy plus trastuzumab in the first-line setting. Chemotherapy plus trastuzumab conferred significantly longer OS, leading to approval of trastuzumab for HER2-positive AGC. Following positive results of new anti-HER 2 drugs for breast cancer, not a few clinical studies of lapatinib[9]9, trastuzumab emtansine [T-DM1] [11-13] and pertuzumab [14] for HER2-positive AGC, including trastuzumab beyond progression [15], were conducted, but none of them elicited a statistically significant improvement in treatment for HER2-positive AGC.

Several mechanisms of resistance may explain these negative results in HER2-positive AGC. First, compared with breast cancer, HER2 staining pattern of AGC is heterogenous, and it is also different between the primary and metastatic lesions [16, 17]. Second, prior trastuzumab treatment 
Table 1 Randomized trials for human epidermal growth factor receptor 2 (HER2) positive advanced gastric cancer

\begin{tabular}{|c|c|c|c|c|c|}
\hline Trial & Phase & Regimen & Line & Median OS (mo) & Median PFS (mo) \\
\hline ToGA $^{8}$ & 3 & $\begin{array}{l}\text { FP/XP + Trastuzumab } \\
\text { FP/XP }\end{array}$ & $1 \mathrm{st}$ & $\begin{array}{l}13.8 \\
11.1 \\
\text { HR } 0.74 ; p=0.0046\end{array}$ & $\begin{array}{l}6.7 \\
5.5 \\
\text { HR } 0.71 ; p=.0002\end{array}$ \\
\hline LOGiC $^{9}$ & 3 & $\begin{array}{l}\text { CapeOX + Lapatinib } \\
\text { CapeOX }\end{array}$ & $1 \mathrm{st}$ & $\begin{array}{l}12.2 \\
10.5 \\
\text { HR } 0.91 ; p=0.3492\end{array}$ & $\begin{array}{l}6.0 \\
5.4 \\
\text { HR } 0.82 ; p=0.0381\end{array}$ \\
\hline $\mathrm{JACOB}^{14}$ & 3 & $\begin{array}{l}\mathrm{XP}+\text { Trastuzumab + Pertuzumab } \\
\mathrm{XP}+\text { Trastuzumab }\end{array}$ & $1 \mathrm{st}$ & $\begin{array}{l}17.5 \\
14.2 \\
\text { HR } 0.84 ; p=0.057\end{array}$ & $\begin{array}{l}8.5 \\
7.0 \\
\text { HR } 0.73 ; p=0.0001\end{array}$ \\
\hline T-ACT ${ }^{15}$ & 2 & $\begin{array}{l}\text { Paclitaxel+ Trastuzumab } \\
\text { Paclitaxel }\end{array}$ & 2 nd & $\begin{array}{l}10.2 \\
10.0 \\
\text { HR } 1.23 ; p=0.20\end{array}$ & $\begin{array}{l}3.7 \\
3.2 \\
\text { HR } 0.91 ; p=0.33\end{array}$ \\
\hline TyTAN $^{10}$ & 3 & $\begin{array}{l}\text { Paclitaxel + Lapatinib } \\
\text { Paclitaxel }\end{array}$ & 2nd & $\begin{array}{l}11.0 \\
8.9 \\
\text { HR } 0.84 ; p=0.1044\end{array}$ & $\begin{array}{l}5.5 \\
4.4 \\
\text { HR } 0.85 ; p=0.2411\end{array}$ \\
\hline GATSBY $^{13}$ & 3 & $\begin{array}{l}\text { T-DM1 } \\
\text { Docetaxel/Paclitaxel }\end{array}$ & $2 \mathrm{nd}$ & $\begin{array}{l}7.9 \\
8.6 \\
\text { HR } 1.15 ; p=0.86\end{array}$ & $\begin{array}{l}2.7 \\
.2 .9 \\
\text { HR } 1.13 ; p=0.31\end{array}$ \\
\hline $\begin{array}{l}\text { DESTINY- } \\
\text { Gas- } \\
\text { tric } 01^{32}\end{array}$ & 2 & $\begin{array}{l}\text { T-DXd } \\
\text { Irinotecan/Paclitaxel }\end{array}$ & $3 \mathrm{rd}$ & $\begin{array}{l}12.5 \\
8.4 \\
\text { HR } 0.59 ; p=0.0097\end{array}$ & $\begin{array}{l}5.6 \\
3.5 \\
\text { HR } 0.47\end{array}$ \\
\hline
\end{tabular}

$X P$, capecitabine and cisplatin; $F P, 5-\mathrm{FU}$ and cisplatin; $C a p e O X$, capecitabine and oxaliplatin; $H R$, hazard ratio; mo, months; OS, overall survival; $P F S$, progression-free survival; T-DM1, trastuzumab emtansine; $T-D X d$, trastuzumab deruxtecan; may lead to a reduction of HER2 expression [18-20]. Finally, tumors may have intrinsic and/or acquired resistant mechanism that reactivates pro-survival pathways such as PI3K/AKT signaling [15, 18, 19]. All these factors pose problems to be resolved for the development of new drugs and/or treatment strategy for anti-HER2 drugs for HER2positive AGC.

\section{Trastuzumab deruxtecan (T-DXd; DS-8201a)}

Antibody-drug conjugates (ADCs) are classified in a new entity of anti-tumor agents. Two ADCs, T-DXd and T-DM1, have been approved for metastatic breast cancer in the United States and Japan. T-DXd is an ADC containing deruxtecan, a topoisomerase I inhibitor, as a payload with a high drug-to-antibody ratio $(8: 1)$, while T-DM1 contains emtansine with a drug-to-antibody ratio of 3.5:1. In T-DXd, the peptide linker is stable in plasma, but after adhesion and internalization into cancer cells, the peptide linker is cleaved by lysosomal enzymes releasing deruxtecan, which has higher anti-tumor activity than irinotecan [21-25]. In contrast, emtansine is less active for GC compared to other malignancies [26], and it has a weaker anti-tumor effect in gastric cancer mouse models than deruxtecan. Noticeably, T-DXd has anti-tumor effects in HER2-negative tumor cells that are close to their HER2-positive counterparts [27, 28]. When internalized T-DXd kills HER2-positive tumor cells, deruxtecan is released into intercellular spaces and then transferred to neighboring HER2-negative tumor cells; so-called bystander killing effect can overcome intra-tumor heterogeneity and reduced HER2 expression (Fig. 1).

T-DXd exhibited anti-tumor efficacy in HER2-positive breast cancer patients $[25,29,30]$ and AGC patients in a phase 1 study [31]. Based on the results of the phase 1 studies, the Food and Drug Adiministration (FDA) approved T-DXd for AGC as breakthrough therapy designation, and the "sakigake" designation scheme was applied in Japan. Subsequently, T-DXd was compared to the chemotherapy of physician's choice (irinotecan or paclitaxel) in the randomized phase 2 trial, DESTINY-Gastric01 [32]. This trial enrolled HER2-positive AGC patients in whom disease had progressed after 2 or more lines of chemotherapy including trastuzumab. The primary endpoint was met, with a significantly higher objective response rate (ORR) $(51.3 \%$ versus $14.3 \%$; $p$ value $<0.0001$ ), prolonged OS (median 12.5 versus 8.4 months; hazard ratio [HR] 0.59; $95 \%$ confidence interval [CI] 0.39-0.88; $p$ value 0.0097) and progression-free survival (PFS) (median5.6 versus 3.5 months; HR 0.47; 95\% CI $0.31-0.71 ; p$ value 0.0003 ). As a result, T-DXd has been approved as salvage-line chemotherapy for HER2-positive AGC in Japan. However, it is necessary to pay attention to adverse events of T-DXd such as grade $\geq 3$ neutropenia (51.2\%), anemia (37.6\%) and thrombocytopenia (11.2\%). Of note, interstitial lung disease (ILD)/pneumonitis occurred in $9.6 \%$ of patients. Most ILD were low grade (9 out of 


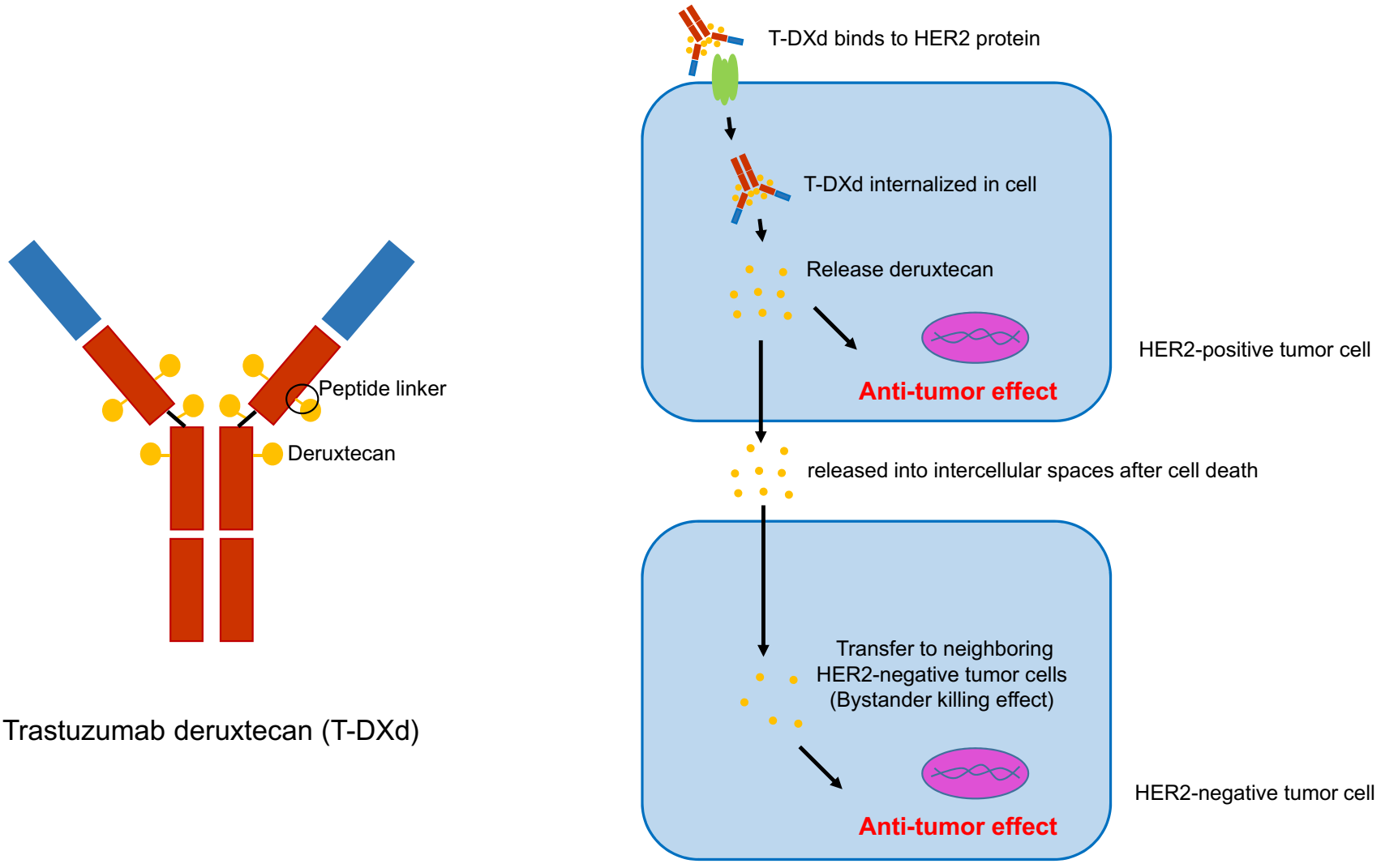

Fig. 1 Graphical overview of trastuzumab deruxtecan (T-DXd). T-DXd is an antibody-drug conjugates (ADC) containing deruxtecan, a topoisomerase I inhibitor, as a payload with a high drug-to-antibody ratio (8:1). In T-DXd, the peptide linker is stable in plasma, but after adhesion to cancer cells, the peptide linker is cleaved by lysosomal

12 patients); grade 3 in 2 patients and grade 4 in 1 patient. ILD was observed less frequently in AGC than breast cancer (13.6\%) [29]. Shorter duration of treatment (4.6 vs 10.0 months) is considered to cause such a lower incidence of ILD even at a higher dose for AGC (6.4 mg/kg vs. $5.4 \mathrm{mg} /$ $\mathrm{kg}$ ). Though data is not published, the history of radiation therapy may be a risk of ILD in breast cancer patients. In addition, considering that irinotecan, an 'earlier generation' topoisomerase I inhibitor, is contraindicated for patients with severe peritoneal metastasis causing bowel passage impairment and/or massive ascites, who are usually excluded from clinical studies. Since T-DXd contains topoisomerase I inhibitor as a payload, the efficacy and safety of T-DXd for such patients must be surveyed in a post-marketing study.

Nowadays, the national comprehensive cancer network (NCCN) guidelines that recommend third- or later-line therapies for AGC are irinotecan [33-36], and trifluridine/ tipiracil (TFTD) [37] regardless HER2 status. Globally, pembrolizumab, an anti-programmed cell-death (PD1) antibody, is used for microsatellite instability-high (MSI-H), deficient mismatch repair (dMMR) [38, 39], or programmed enzymes releasing deruxtecan. When internalized T-DXd kills human epidermal growth factor receptor 2 (HER2) positive tumor cells, deruxtecan is released into intercellular spaces and then transferred to neighboring HER2-negative tumor cells; so-called bystander killing effect

cell-death ligand 1 (PD-L1) combined positive score (CPS) $\geq 1$ AGC [40]. In the Japanese Gastric Cancer Treatment Guideline, nivolumab is recommended without specific indication as the third- or later-line treatment, according to the results from the ATTRACTION-2 trial [41]. Although the trial for T-DXd (DESTINY-Gastric01) included only HER-2 positive AGC and efficacy data of other drugs for HER2-positive AGC are not fully available, the results of the four studies for the third-line or subsequent setting are summarized in Table 2 including the sub-population with prior use of trastuzumab in the ATTRACTION-2 trial [42]. While nivolumab showed slightly better efficacy for patients with prior use of trastuzumab than others, there have been no reports showing different efficacy of irinotecan and TFTD according to the HER2 status. As for irinotecan, 55 of 62 patients in the control arm of the DESTINY-Gastric01 trial received irinotecan by physician's choice although there is no prospective study of it in the third-line or later setting. Among these four drugs, T-DXd appears to elicit the most favorable response, PFS and OS in third- or later line settings for HER2-positive AGC than other drugs. Thus, it is 
Table 2 Phase 2 or 3 trials for advanced gastric cancer in third- or later-line settings

\begin{tabular}{|c|c|c|c|c|c|c|}
\hline Trial & Phase & Compound & HER2 status & Median OS (mo) & Median PFS (mo) & ORR $(\%)$ \\
\hline TAGS $^{37}$ & 3 & TFTD & Not required & $\begin{array}{l}5.7 \\
(95 \% \text { CI } 4.8-6.2)\end{array}$ & $\begin{array}{l}2.0 \\
(95 \% \text { CI } 1.9-2.3)\end{array}$ & $\begin{array}{l}4.0 \\
(95 \% \text { CI 2-8) }\end{array}$ \\
\hline KEYNOTE- $059^{40}$ & 2 & Pembrolizumab & Not required & $\begin{array}{l}5.6 \\
(95 \% \text { CI 4.3-6.9) }\end{array}$ & $\begin{array}{l}2.0 \\
(95 \% \text { CI } 2.0-2.1)\end{array}$ & $\begin{array}{l}11.6 \\
(95 \% \text { CI } 8.0-16.1) \\
15.5 * 1 \\
(95 \% \text { CI } 10.1-22.4)\end{array}$ \\
\hline Attraction- $2^{41}$ & 3 & Nivolumab & Not required & $\begin{array}{l}5.3 \\
(95 \% \text { CI } 4.6-6.4)\end{array}$ & $\begin{array}{l}1.6 \\
(95 \% \text { CI } 1.5-2.3)\end{array}$ & $\begin{array}{l}11.2 \\
(95 \% \text { CI } 7.7-15.6)\end{array}$ \\
\hline $\begin{array}{l}\text { Attraction- } 2 \\
\text { subgroup analysis } 42 * 2\end{array}$ & 3 & Nivolumab & Not required & $\begin{array}{l}8.3 \\
(95 \% \text { CI } 5.3-12.9)\end{array}$ & $\begin{array}{l}1.6 \\
(95 \% \text { CI } 1.5-4.0)\end{array}$ & 16.9 \\
\hline \multirow[t]{2}{*}{ DESTINY-Gastric01 ${ }^{32}$} & 2 & T-DXd & Required & $\begin{array}{l}12.5 \\
(95 \% \text { CI } 9.6-14.3)\end{array}$ & $\begin{array}{l}5.6 \\
(95 \% \text { CI 4.3-6.9) }\end{array}$ & $\begin{array}{l}51.3 \\
(95 \% \text { CI 41.9-60.5) }\end{array}$ \\
\hline & & $\begin{array}{l}\text { CPT-11 }(\mathrm{n}=55) \\
\operatorname{PTX}(\mathrm{n}=7)\end{array}$ & Required & $\begin{array}{l}8.4 \\
(95 \% \text { CI 6.9-10.7) }\end{array}$ & $\begin{array}{l}3.5 \\
(95 \% \text { CI } 2.0-4.3)\end{array}$ & $\begin{array}{l}14.3 \\
(95 \% \text { CI 6.4-26.2) }\end{array}$ \\
\hline
\end{tabular}

$C I$, confidence interval; HER2, human epidermal growth factor receptor 2; mo, months; ORR, objective response rate; OS, overall survival; PFS, progression-free survival; $T$ - DXd, trastuzumab deruxtecan; TFTD, Trifluridine/tipiracil; CPT-11, irinotecan; PTX, paclitaxel

*1 Limited to patients with programmed cell-death ligand 1 (PD-L1)-positive

*2 Limited to patients with prior use of trastuzumab

considered that T-DXd can be recommended as the first choice as third-line chemotherapy for HER2-positive AGC.

\section{Development of combination therapy with other anti-cancer agents}

In the future, the replacement of trastuzumab by T-DXd in combination with other anti-cancer agents is expected (Table 3). However, the safety of T-DXd in combination with cytotoxic agents is a big concern since T-DXd induces substantial hematological toxicity. Considering about different recommended doses of T-DXd for breast cancer $(5.4 \mathrm{mg} /$ $\mathrm{kg})$ and for gastric cancer $(6.4 \mathrm{mg} / \mathrm{kg}), \mathrm{T}-\mathrm{DXd}$ can be used at $5.4 \mathrm{mg} / \mathrm{kg}$ or lower in combination with cytotoxic agents. The DESTINY-Breast07 (NCT04538742) will start recruiting soon, and the safety results of T-DXd in combination with durvalumab, paclitaxel and pertuzumab will be a reference for other combination with cytotoxic agents.

Chemoradiation therapy is one treatment option especially for gastroesophageal junction cancer, which shows higher HER2-positive rates than GC (33.2\% vs. 20.9\%) [43], although its indication is generally limited to the locally advanced cases without distant metastasis. Overexpression of HER2 and its downstream PI3K/Akt pathway plays a role in resistance to radiation therapy $[44,45]$. A preclinical study demonstrated that trastuzumab enhanced sensitivity to radiation therapy in breast cancer cell lines [44, 46]. In clinical studies, the combination of HER2-targeted therapy and radiation therapy was well tolerated and elicited a favorable outcome in breast cancer [47-49]. While trastuzumab in combination with chemoradiation therapy was well tolerated in TOXAG trial [50], the conjugated topoisomerase I inhibitor payload of T-DXd has been associated with a risk of pneumonitis. Indeed, in the

Table 3 Expected development of combination therapy with other anti-cancer agents

Cytotoxic agents

T-Dxd has higher anti-tumor activity than trastuzumab

Safety of T-DXd in combination with cytotoxic agents is a big concern

Chemoradiation therapy

Overexpression of HER2 and its downstream PI3K/Akt pathway play a role for resistance to radiation therapy

Molecular-targeted drugs

HER2 signaling increases VEGF

Activation of PI3K/AKT signaling is a mechanism of resistance to HER2-targeted therapy

Immunotherapy

HER2 signaling is immunosuppressive and HER2-targeted therapy leads to increase numbers of TILs

$H E R 2$, human epidermal growth factor receptor 2; T-DXd, trastuzumab deruxtecan; TILs, tumor-infiltrating lymphocytes; VEGF, vascular endothelial growth factor; VEGFR-2, vascular endothelial growth factor receptor 2 
DESTINY-Gastric01 trial, ILD/pneumonitis occurred in 9.6\% of patients. Thus, combining T-DXd with radiation is expected to be efficacious for HER-2 positive locally advanced gastroesophageal junction cancer, but pneumonitis must be monitored carefully and managed appropriately.

Considering combinations with other molecular-targeted drugs for HER2-positive AGC patients, ramucirumab, an antivascular endothelial growth factor receptor 2 (VEGFR-2) antibody, is the only approved molecular-targeted agent besides trastuzumab at present. In a preclinical study, a HER2 signaling-dependent increase in vascular endothelial growth factor (VEGF) was reported [51], and targeting both HER2 and VEGF enhanced tumor growth inhibition in a breast cancer mouse model [52]. In a clinical trial of breast cancer, a combination of an anti-HER2 agent and anti-VEGF-antibody bevacizumab was safe and showed promising efficacy [53, 54]. Furthermore, activation of PI3K/AKT signaling is a mechanism of resistance to HER2-targeted therapy, and targeted inhibition of this pathway could be expected to be effective. However, mTOR inhibitor everolimus alone was not so effective in AGC patients. For breast cancer, everolimus with trastuzumab and paclitaxel prolonged PFS than placebo with trastuzumab and paclitaxel in hormone receptor-negative, HER2-positive population [55]. Thus, other molecular-targeted agents such as everolimus should be evaluated in combination with T-DXd.

Combination of HER2 inhibitors with immunotherapy is also expected to have synergic efficacy because activation of HER2 signaling is reported to be immunosuppressive[56] and HER2-targeted therapy leads to increased numbers of tumorinfiltrating lymphocytes (TILs) [57, 58]. Preclinical data show that HER2-targeted therapy induces type I and II interferons and synergizes with an immune checkpoint inhibitors [59]. In a murine xenograft model, a combination of T-DXd with antiPD-1 antibody provided more efficacy than T-DXd alone [60]. In the PANACEA phase Ib/II study, pembrolizumab plus trastuzumab suggested clinical benefits in patients with HER 2 and PD-L1-positive breast cancer [61]. Furthermore, combining VEGF blockade with immunotherapy also activates T cells. In AGC patients, a combination of nivolumab and ramucirumab exhibits antitumor activity (NCT02999295) [62]. Thus, a combination of T-DXd with immunotherapy plus ramucirumab can be considered.

\section{Potential treatment strategies using T-DXd (Fig. 2)}

\section{First-line setting}

DESTINY-Gastric03 recruits AGC patients in the firstline setting (Table 4), which investigates the safety and anti-tumor activity of various regimens using T-DXd in combination with other cytotoxic agents (single or combination of 5-FU/capecitabine and cisplatin/oxaliplatin) and/or immune checkpoint inhibitor (durvalumab). It is expected that T-DXd will replace trastuzumab and show better efficacy when combined with doublet chemotherapy as the first-line treatment for AGC. The addition of T-DXd to platinum and fluoropyrimidines is considered feasible since it mimics the triplet chemotherapy of 5-FU, oxaliplatin, and irinotecan that is widely used for colorectal and pancreatic cancers. Although adverse events may be a concern, T-DXd is potentially more powerful for HER2positive AGC patients. To mitigate adverse effects, for breast cancer, 3 drugs for neoadjuvant chemotherapy are sequentially used (doxorubicin plus cyclophosphamide followed by docetaxel). Also, the ORR of T-DXd (51.3\% in DESTINY-Gastric01 trail) was as high as platinum plus fluoropyrimidines (54\% in SPIRITS trail [63]) even though DESTINY-Gastric01 trail was third- or later line treatment. Therefore, it is expected that triplet chemotherapy with sequential use of T-DXd (T-DXd followed by platinum and fluoropyrimidines or its reverse sequence) can enhance the power of chemotherapy compared with doublet regimen.

In 2020, it has been demonstrated that the addition of nivolumab to doublet chemotherapy with fluoropyrimidine plus platinum provides a survival benefit for HER2-negative AGC patients $[64,65]$, and the addition of nivolumab to doublet chemotherapy with 5-FU plus irinotecan for gastroesophageal cancer patients showed promising efficacy [66]. Immune checkpoint inhibitors are expected to have a survival benefit in the first-line therapy also for HER2-positive AGC. Pembrolizumab in combination with trastuzumab and capecitabine plus oxaliplatin in the firstline setting elicited a high ORR (83\%) in a phase 2 study in HER2 positive esophagogastric adenocarcinoma [67]. Also, first-line treatment of AGC patients with pembrolizumab in combination with trastuzumab and chemotherapy (5-FU plus cisplatin, S-1 plus oxaliplatin, or capecitabine plus oxaliplatin) is currently evaluated in a phase 3 trial (KEYNOTE-811; NCT03615326). Thus, if the KEYNOTE-811 trial is positive, the next challenge will be the replacement of trastuzumab by T-DXd in combination with cytotoxic and immunological agents.

\section{Second-line setting}

DESTINY-Gastric02 (NCT04014075), is an ongoing single-arm phase 2 study of T-DXd in the second-line setting for HER2-positive AGC which progressed after trastuzumab. Also phase 3 study in the second-line setting DESTINY-Gastric04 (NCT04704934), patients receive T-DXd or standard regimen (paclitaxel plus ramucirumab), will 


\section{Standard chemotherapy recommended at present and T-DXd containing regimens expected in future for HER2-positive AGC}

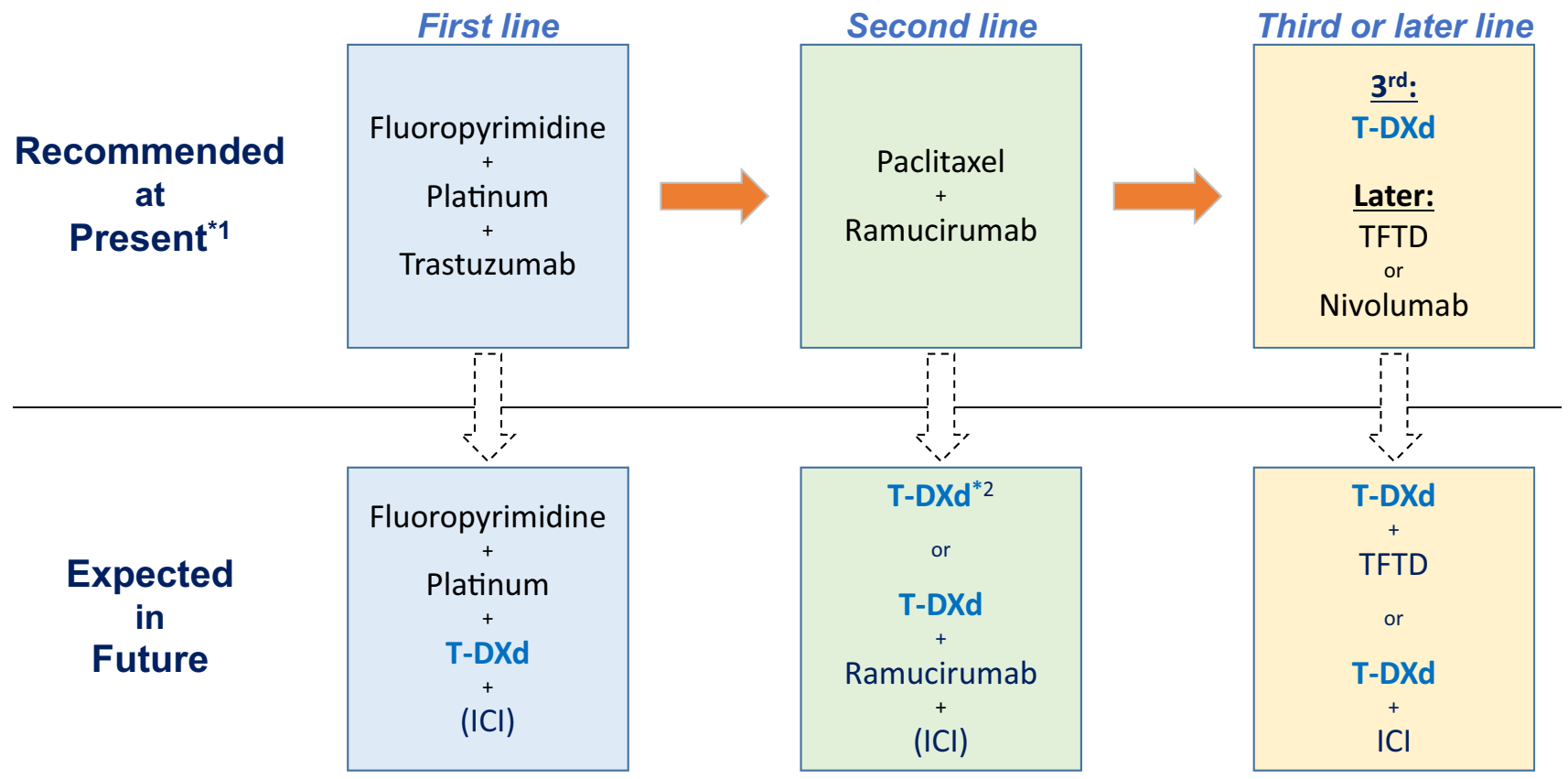

Fig. 2 Illustration of the standard chemotherapy recommended at present and T-DXd containing regimens expected in the future for HER2-positive AGC. T-DXd is expected to establish a new standard treatment for HER2-positive AGC: (1) replacement of trastuzumab with/out immune checkpoint inhibitor in the first-line setting, (2) replacement of paclitaxel in combination with ramucirumab, or paclitaxel plus ramucirumab with/out immune checkpoint inhibitor in the second-line setting (3) combination with trifluridine/tipiracil or immune checkpoint inhibitor. *1: modification of the Japanese Gastric Cancer Treatment Guideline, *2: A phase III study (DESTINYGastric04) will start soon. TFTD trifluridine/tipiracil, ICI immune checkpoint inhibitor
Table 4 Arms and regimens of phase $1 \mathrm{~b} / 2$ study DESTINYGastric03

\begin{tabular}{ll}
\hline Arm & Regimen \\
\hline $\begin{array}{l}\text { Experimental: } \\
\text { Arm 1A }\end{array}$ & T-DXd and 5-FU \\
Experimental: & T-DXd and capecitabine \\
Arm 1B & \\
Experimental: & T-DXd and durvalumab \\
Arm 1C & T-DXd and 5-FU or capecitabine and oxaliplatin \\
$\begin{array}{l}\text { Experimental: } \\
\text { Arm 1D }\end{array}$ & T-DXd, durvalumab and 5-FU or capecitabine \\
$\begin{array}{l}\text { Experimental: } \\
\text { Arm 1E }\end{array}$ & Trastuzumab, 5-FU/capecitabine, and cisplatin/oxaliplatin \\
$\begin{array}{l}\text { Active Comparator: } \\
\text { Arm 2A }\end{array}$ & \\
$\begin{array}{l}\text { Experimental: } \\
\text { Arm 2B }\end{array}$ & T-DXd monotherapy \\
$\begin{array}{l}\text { Experimental: } \\
\text { Arm 2C }\end{array}$ & T-DXd, 5-FU or capecitabine, and oxaliplatin \\
$\begin{array}{l}\text { Experimental: } \\
\text { Arm 2D }\end{array}$ & T-DXd, 5-FU or capecitabine, and durvalumab \\
\hline
\end{tabular}

$T$-DXd, trastuzumab deruxtecan 
start recruiting. In the RAINBOW study, paclitaxel plus ramucirumab was efficacious and produced a median PFS of 4.4 months (95\% CI 4.2-5.3) and an ORR of $28 \%$ (95\% CI 23-33) [68]. Adding T-DXd to this regimen could be a powerful strategy; however, both paclitaxel and T-DXd are associated with a risk of ILD/pneumonitis and hematological toxicities. T-DXd provided a survival benefit for HER2-positive AGC patients over the physician's choice chemotherapy, mainly irinotecan, in the third-line setting. In the WJOG 4007 trial, irinotecan showed equivalent efficacy to paclitaxel in second-line chemotherapy for AGC patients [33]. Considering the synergic effects with antiangiogenic agents shown in basic research, it is expected that T-DXd will show superiority to paclitaxel in combination with ramucirumab as the second-line therapy for HER2-positive AGC patients. Moreover, there is more availability of subsequent chemotherapy after T-DXd plus ramucirumab because paclitaxel is feasible even for AGC patients with severe peritoneal metastasis in third- or later line setting for which irinotecan is contraindicated. Thus, considering the points of greater efficacy of T-DXd and availability of subsequent chemotherapy, treatment sequence T-DXd plus ramucirumab followed by paclitaxel is expected to prolong the survival of AGC patients.

Early recurrence during or shortly after peri-operative chemotherapy is another situation of second-line treatment, and same agents used in perioperative chemotherapy are reported to show low efficacy after recurrence. In the NCCN guidelines, the preferred regimens for perioperative chemotherapy are doublet with fluoropyrimidine and oxaliplatin or triplet with fluorouracil, leucovorin, oxaliplatin and docetaxel (FLOT). In Japan, S-1 plus docetaxel is one of standard adjuvant chemotherapy for stage III curatively resected GC [69]. In this situation of HER2 positive recurrent GC, platinum, irinotecan, ramucirumab and trastuzumab are available, now a phase II trial (OGSG1901) of irinotecan plus ramucirumab for early recurrence during or shortly after adjuvant chemotherapy with S-1 plus docetaxel is ongoing. In the near future, it is expected that HER2-targeted therapy can be included in chemotherapy for early recurrence in HER2-positive AGC patients (e.g. T-DXd plus ramucirumab).

\section{Third-line setting}

Since irinotecan is replaced by T-DXd based on the results of DESTINY-Gastric01 trial [32] after the failure of two lines of standard chemotherapy using fluoropyrimidine, platinum and taxane, there are currently three agents available for the third-line chemotherapy of HER2-positive AGC: immune checkpoint inhibitors, TFTD and T-DXd. It is generally recognized that all available drugs should be used throughout the whole treatment course. However, few patients maintain sufficient fitness after the failure of the third-line chemotherapy. Moreover, many patients with severe peritoneal metastasis are not suitable for treatment with oral agents and irinotecan. In our case series at the National Cancer Center Hospital, about $50 \%$ of patients receiving the third-line chemotherapy could receive the fourth-line chemotherapy, accounting for less than $30 \%$ of all AGC patients (data not shown). Thus, the development of more powerful combination chemotherapies with low toxicity using these agents is warranted in the third-line setting. As mentioned above, combination of T-DXd with immunotherapy is promising. There are clinical studies of T-DXd with immune checkpoint inhibitors; nivolumab for breast and urothelial cancer (NCT03523572), and pembrolizumab for breast and nonsmall cell lung cancer (NCT04042701). Since HER2-positive AGC patients were not included in the Checkmate-649 (NCT02872116) and ATTRACTION-4 (NCT02746796) trials, which showed benefits of nivolumab for HER2 negative AGC in the first line, most HER-2 positive patients with prior use of trastuzumab are nivolumab-naïve. Combination of T-DXd with immune checkpoint inhibitor is a promising candidate in the third-line setting for HER2-positive AGC.

Another candidate is the combination of T-DXd with TFTD. In a mouse models, a combination of TFTD and irinotecan had significantly higher antitumor activity than monotherapy [70]. One component of TFTD, trifluridine, is incorporated into DNA. This genotoxic mechanism of action may synergize with the DNA strand breakage that is induced by T-DXd-dependent inhibition of topoisomerase I. Furthermore, combination chemotherapy with these all three drugs can be considered.

\section{Perioperative setting}

Perioperative chemotherapy incorporating HER-targeted agents is well established for the treatment of HER2-positive breast cancer, which is currently under investigation for GC. INNOVATION (NCT02205047) is a phase 2 study to evaluate the efficacy of trastuzumab and pertuzumab in the perioperative setting in HER2-positive GC patients. T-DXd is also expected to be efficacious in the perioperative setting. As mentioned above, however, the current global standard perioperative treatment is triplet chemotherapy, and toxicities by adding T-DXd is a great concern. Substituting T-DXd for one of the cytotoxic agents in the present standard chemotherapy regimen such as docetaxel is an option. Instead, immunotherapy can be added. In melanoma, adjuvant therapy with nivolumab led to significantly longer recurrencefree survival [71]. In breast cancer, neoadjuvant therapy with pembrolizumab plus chemotherapy was associated with a significantly higher pathological CR than chemotherapy alone [72]. In GC, a trial to evaluate perioperative chemotherapy with pembrolizumab is ongoing (NCT03221426). 
Nivolumab or pembrolizumab combined with T-DXd is another candidate perioperative chemotherapy for HER2positive GC.

\section{Low HER2 expression}

T-DXd inhibited tumor growth in mice xenografts with various levels of HER2 expression [25]. In a recent clinical study, T-DXd was efficacious in breast cancer patients whose tumors had low HER 2 expression (IHC $2+$ and ISH negative or IHC $1+$ )[30]. In the DESTINY-Gastric01 study, patients with IHC $2+$ and ISH (-) and those with IHC $1+$ were also included in the exploratory cohort [73]. The confirmed ORR in each of these groups was $26.3 \%$ and $9.5 \%$, respectively. Even though the effect was weaker than in high HER2strongly positive patients, these data show that T-DXd has substantial activity even in HER2-low AGC. Thus, T-DXd has a potential to extend its indication to HER2-low AGC in all clinical settings mentioned above.

\section{Conclusions}

For HER2-positive AGC, T-DXd has been introduced to clinical practice as salvage line chemotherapy for HER2positive AGC. In the future, T-DXd will contribute to advances in the treatment of patients with HER2-positive AGC in various clinical settings.

\section{References}

1. Slamon DJ, Clark GM, Wong SG, Levin WJ, Ullrich A, McGuire WL. Human breast cancer: correlation of relapse and survival with amplification of the HER-2/neu oncogene. Science. 1987;235(4785):177-82.

2. Slamon DJ, Leyland-Jones B, Shak S, Fuchs H, Paton V, Bajamonde A, et al. Use of chemotherapy plus a monoclonal antibody against HER 2 for metastatic breast cancer that overexpresses HER2. N Engl J Med. 2001;344(11):783-92.

3. Bray F, Ferlay J, Soerjomataram I, Siegel R, Torre L, Jemal A. Global cancer statistics 2018: GLOBOCAN estimates of incidence and mortality worldwide for 36 cancers in 185 countries. CA Cancer J Clin. 2018;68(6):394-424.

4. Gravalos C, Jimeno A. HER2 in gastric cancer: a new prognostic factor and a novel therapeutic target. Ann Oncol. 2008;19:1523-9.

5. Bartley AN, Washington MK, Colasacco C, Ventura CB, Ismaila $\mathrm{N}$, Benson $\mathrm{AB}$ 3rd, et al. HER2 testing and clinical decision making in gastroesophageal adenocarcinoma: guideline from the college of American Pathologists, American Society for Clinical Pathology, and the American Society of Clinical Oncology. J Clin Oncol. 2017;35:446-64.

6. Fuse N, Kuboki Y, Kuwata T, Nishina T, Kadowaki S, Shinozaki E, et al. Prognostic impact of HER2, EGFR, and c-MET status on overall survival of advanced gastric cancer patients. Gastric Cancer. 2015;19:183-91.

7. Aizawa M, Nagatsuma AK, Kitada K, Kuwata T, Fujii S, Kinoshita T, et al. Evaluation of HER2-based biology in 1,006 cases of gastric cancer in a Japanese population. Gastric Cancer. 2014; 17:34-42.

8. Bang Y-J, Van Cutsem E, Feyereislova A, Chung HC, Shen L, Sawaki A, et al. Trastuzumab in combination with chemotherapy versus chemotherapy alone for treatment of HER2-positive advanced gastric or gastro-oesophageal junction cancer (ToGA): a phase 3, open-label, randomised controlled trial. Lancet. 2010;376:687-97.

9. Hecht JR, Bang YJ, Qin SK, Chung HC, Xu JM, Park JO, et al. Lapatinib in combination with capecitabine plus oxaliplatin in human epidermal growth factor receptor 2-positive advanced or metastatic gastric, esophageal, or gastroesophageal adenocarcinoma: TRIO-013/LOGiC-a randomized phase III trial. J Clin Oncol. 2016;34:443-51.

10. Satoh T, Xu RH, Chung HC, Sun GP, Doi T, Xu JM, et al. Lapatinib plus paclitaxel versus paclitaxel alone in the second-line treatment of HER2-amplified advanced gastric cancer in Asian populations: TyTAN--a randomized, phase III study. J Clin Oncol. 2014;32:2039-49.

11. Junttila TT, Li G, Parsons K, Phillips GL, Sliwkowski MX. Trastuzumab-DM1 (T-DM1) retains all the mechanisms of action of trastuzumab and efficiently inhibits growth of lapatinib insensitive breast cancer. Breast Cancer Res Treat. 2011;128:347-56.

12. Lewis Phillips GD, Li G, Dugger DL, Crocker LM, Parsons KL, Mai E, et al. Targeting HER2-positive breast cancer with trastuzumab-DM1, an antibody-cytotoxic drug conjugate. Cancer Res. 2008;68:9280-90.

13. Thuss-Patience PC, Shah MA, Ohtsu A, Van Cutsem E, Ajani JA, Castro $\mathrm{H}$, et al. Trastuzumab emtansine versus taxane use for previously treated HER2-positive locally advanced or metastatic gastric or gastro-oesophageal junction adenocarcinoma (GATSBY): an international randomised, open-label, adaptive, phase 2/3 study. Lancet Oncol. 2017;18:640-53.

14. Tabernero J, Hoff PM, Shen L, Ohtsu A, Shah MA, Cheng K, et al. Pertuzumab plus trastuzumab and chemotherapy for HER2positive metastatic gastric or gastro-oesophageal junction cancer (JACOB): final analysis of a double-blind, randomised, placebocontrolled phase 3 study. Lancet Oncol. 2018;19:1372-84.

15. Makiyama A, Sukawa Y, Kashiwada T, Kawada J, Hosokawa A, Horie Y, et al. Randomized, phase II study of trastuzumab beyond progression in patients with HER2-positive advanced gastric or gastroesophageal junction cancer: WJOG7112G (T-ACT Study). J Clin Oncol. 2020;38(17):1919-27.

16. Ieni A, Barresi V, Caltabiano R, Caleo A, Bonetti LR, Lanzafame $\mathrm{S}$, et al. Discordance rate of HER2 status in primary gastric carcinomas and synchronous lymph node metastases: a multicenter retrospective analysis. Int J Mol Sci. 2014;15:22331-41.

17. Gumusay O, Benekli M, Ekinci O, Baykara M, Ozet A, Coskun $\mathrm{U}$, et al. Discordances in HER2 status between primary gastric cancer and corresponding metastatic sites. Jpn J Clin Oncol. 2015;45:416-21.

18. Janjigian YY, Sanchez-Vega F, Jonsson P, Chatila WK, Hechtman JF, Ku GY, et al. Genetic predictors of response to systemic therapy in esophagogastric cancer. Cancer Discov. 2018;8:49-58.

19. Wang DS, Liu ZX, Lu YX, Bao H, Wu X, Zeng ZL, et al. Liquid biopsies to track trastuzumab resistance in metastatic HER2positive gastric cancer. Gut. 2019;68:1152-61.

20. Pietrantonio F, Caporale M, Morano F, Scartozzi M, Gloghini A, De Vita F, et al. HER2 loss in HER2-positive gastric or gastroesophageal cancer after trastuzumab therapy: implication for further clinical research. Int J Cancer. 2016;139(12):2859-64. 
21. Shiose Y, Ochi Y, Kuga H, Yamashita F, Hashida M. Relationship between drug release of DE-310, macromolecular prodrug of DX8951f, and cathepsins activity in several tumors. Biol Pharm Bull. 2007;30(12):2365-70.

22. Niedergethmann M, Wostbrock B, Sturm J, Willeke F, Post S, Hildenbrand R. Prognostic impact of cysteine proteases cathep$\sin \mathrm{B}$ and cathepsin L in pancreatic adenocarcinoma. Pancreas. 2004;29(3):204-11.

23. Aggarwal N, Sloane BF. Cathepsin B: multiple roles in cancer. Proteom Clin Appl. 2014;8:427-37.

24. Mohamed MM, Sloane BF. Cysteine cathepsins: multifunctional enzymes in cancer. Nat Rev Cancer. 2006;6:764-75.

25. Ogitani Y, Aida T, Hagihara K, Yamaguchi J, Ishii C, Harada $\mathrm{N}$, et al. DS-8201a, A novel HER2-targeting ADC with a novel DNA topoisomerase I inhibitor, demonstrates a promising antitumor efficacy with differentiation from T-DM1. Clin Cancer Res. 2016;22:5097-108.

26. Matthew HK, Alona M, Jeffrey C, Peter CE, Panos F, Kate K, et al. A Phase II trial of vinorelbine in patients with advanced gastroesophageal adenocarcinoma. Cancer Invest. 2006;24(4):346-50.

27. Ogitani Y, Hagihara K, Oitate M, Naito H, Agatsuma T. Bystander killing effect of DS-8201a, a novel anti-human epidermal growth factor receptor 2 antibody-drug conjugate, in tumors with human epidermal growth factor receptor 2 heterogeneity. Cancer Sci. 2016;107:1039-46.

28. Takegawa N, Tsurutani J, Kawakami H, Yonesaka K, Kato R, Haratani K, et al. [fam-] trastuzumab deruxtecan, antitumor activity is dependent on HER2 expression level rather than on HER2 amplification. Int J Cancer. 2019;145(12):3414-24.

29. Modi S, Saura C, Yamashita T, Park YH, Kim SB, Tamura K, et al. Trastuzumab deruxtecan in previously treated HER2-positive breast cancer. N Engl J Med. 2020;382:610-21.

30. Modi S, Park H, Murthy R, Iwata H, Tamura K, Tsurutani J, et al. Antitumor activity and safety of trastuzumab deruxtecan in patients with HER2-low-expressing advanced breast cancer: results from a phase Ib study. J Clin Oncol 2020; 38(17):1887-96.

31. Shitara K, Iwata H, Takahashi S, Tamura K, Park H, Modi S, et al. Trastuzumab deruxtecan (DS-8201a) in patients with advanced HER2-positive gastric cancer: a dose-expansion, phase 1 study. Lancet Oncol. 2019;20:827-36.

32. Shitara K, Bang Y, Iwasa S, Sugimoto N, Ryu M, Sakai D, et al. Trastuzumab Deruxtecan in previously treated HER2-positive gastric cancer. N Engl J Med. 2020;382(25):2419-30.

33. Hironaka $S$, Ueda $S$, Yasui $H$, Nishina $T$, Tsuda $M$, Tsumura $T$, et al. Randomized, open-label, phase III study comparing irinotecan with paclitaxel in patients with advanced gastric cancer without severe peritoneal metastasis after failure of prior combination chemotherapy using fluoropyrimidine plus platinum: WJOG 4007 trial. J Clin Oncol. 2013;31:4438-44.

34. Sym SJ, Hong J, Park J, Cho EK, Lee JH, Park YH, et al. A randomized phase II study of biweekly irinotecan monotherapy or a combination of irinotecan plus 5-fluorouracil/leucovorin (mFOLFIRI) in patients with metastatic gastric adenocarcinoma refractory to or progressive after first-line chemotherapy. Cancer Chemother Pharmacol. 2013;71:481-8.

35. Thuss-Patience PC, Kretzschmar A, Bichev D, Deist T, Hinke A, Breithaupt K, et al. Survival advantage for irinotecan versus best supportive care as second-line chemotherapy in gastric cancer-a randomised phase III study of the Arbeitsgemeinschaft Internistische Onkologie (AIO). Eur J Cancer. 2011;47:2306-14.

36. Fuchs CS, Moore MR, Harker G, Villa L, Rinaldi D, Hecht JR. Phase III comparison of two irinotecan dosing regimens in second-line therapy of metastatic colorectal cancer. J Clin Oncol. 2003;21:807-14.

37. Shitara K, Doi T, Dvorkin M, Mansoor W, Arkenau H-T, Prokharau A, et al. Trifluridine/tipiracil versus placebo in patients with heavily pretreated metastatic gastric cancer (TAGS): a randomised, double-blind, placebo-controlled, phase 3 trial. Lancet Oncol. 2018;19:1437-48.

38. Le DT, Durham JN, Smith KN, Wang H, Bartlett BR, Aulakh LK, et al. Mismatch repair deficiency predicts response of solid tumors to PD-1 blockade. Science. 2017;357:409-13.

39. Le DT, Uram JN, Wang H, Bartlett BR, Kemberling H, Eyring $\mathrm{AD}$, et al. PD-1 blockade in tumors with mismatch-repair deficiency. N Engl J Med. 2015;372:2509-20.

40. Fuchs CS, Doi T, Jang RW, Muro K, Satoh T, Machado M, et al. Safety and efficacy of pembrolizumab monotherapy in patients with previously treated advanced gastric and gastroesophageal junction cancer: phase 2 clinical KEYNOTE-059 trial. JAMA Oncol. 2018;4:e180013.

41. Kang Y-K, Boku N, Satoh T, Ryu M-H, Chao Y, Kato K, et al. Nivolumab in patients with advanced gastric or gastro-oesophageal junction cancer refractory to, or intolerant of, at least two previous chemotherapy regimens (ONO-4538-12, ATTRAC TION-2): a randomised, double-blind, placebo-controlled, phase 3 trial. Lancet. 2017;390:2461-71.

42. Satoh T, Kang YK, Chao Y, Ryu MH, Kato K, Cheol Chung H, et al. Exploratory subgroup analysis of patients with prior trastuzumab use in the ATTRACTION-2 trial: a randomized phase III clinical trial investigating the efficacy and safety of nivolumab in patients with advanced gastric/gastroesophageal junction cancer. Gastric Cancer. 2020;23:143-53.

43. Bang Y, Chung H, Xu J, Lordick F, Sawaki A, Al-Sakaff N, et al. Pathological features of advanced gastric cancer $(\mathrm{GC})$ : relationship to human epidermal growth factor receptor 2 (HER2) positivity in the global screening programme of the ToGA trial. J Clin Oncol. 2009;27(15s:suppl). (abstr 4556).

44. Liang K, Lu Y, Jin W, Kian Ang K, Milas L, Fan Z. Sensitization of breast cancer cells to radiation by trastuzumab. Mol Cancer Ther. 2003;2(11):1113-20.

45. Guo G, Wang T, Gao Q, Tamae D, Wong P, Chen T, et al. Expression of ErbB2 enhances radiation-induced NF-kappaB activation. Oncogene. 2004;23:535-45.

46. Adams SR, Yang HC, Savariar EN, Aguilera J, Crisp JL, Jones $\mathrm{KA}$, et al. Anti-tubulin drugs conjugated to anti-ErbB antibodies selectively radiosensitize. Nat Commun. 2016;7:13019.

47. Halyard MY, Pisansky TM, Dueck AC, Suman V, Pierce L, Solin L, et al. Radiotherapy and adjuvant trastuzumab in operable breast cancer: tolerability and adverse event data from the NCCTG Phase III Trial N9831. J Clin Oncol. 2009;27:2638-44.

48. Horton JK, Halle J, Ferraro M, Carey L, Moore DT, Ollila D, et al. Radiosensitization of chemotherapy-refractory, locally advanced or locally recurrent breast cancer with trastuzumab: a phase II trial. Int J Radiat Oncol Biol Phys. 2010;76:998-1004.

49. Jacob J, Belin L, Pierga JY, Gobillion A, Vincent-Salomon A, Dendale R, et al. Concurrent administration of trastuzumab with locoregional breast radiotherapy: long-term results of a prospective study. Breast Cancer Res Treat. 2014;148:345-53.

50. Abali H, Yalcin S, Onal C, Dane F, Oksuzoglu B, Ozdemir N, et al. A study of the combination of oxaliplatin, capecitabine, and trastuzumab and chemo-radiotherapy in the adjuvant setting in operated patients with HER2+ gastric or gastroesophageal junction cancer (TOXAG study). J Clin Oncol. 2018;36(4s:suppl). (abstr 26).

51. Laughner E, Taghavi P, Chiles K, Mahon PC, Semenza GL. HER2 (neu) signaling increases the rate of hypoxia-inducible factor 1alpha (HIF-1alpha) synthesis: novel mechanism for HIF1-mediated vascular endothelial growth factor expression. Mol Cell Biol. 2001;21:3995-4004.

52. Xiao-Feng L, Weiqun M, Chunhua L, Angela T, John VH, Anil $\mathrm{KS}$, et al. Specific blockade of VEGF and HER2 pathways results 
in greater growth inhibition of breast cancer xenografts that overexpress HER2*. Cell Cycle. 2008;7(23):3747-58.

53. Martin M, Makhson A, Gligorov J, Lichinitser M, Lluch A, Semiglazov V, et al. Phase II study of bevacizumab in combination with trastuzumab and capecitabine as first-line treatment for HER2 -positive locally recurrent or metastatic breast cancer. Oncologist. 2012;17:469-75.

54. Pierga J-Y, Petit T, Delozier T, Ferrero J-M, Campone M, Gligorov J, et al. Neoadjuvant bevacizumab, trastuzumab, and chemotherapy for primary inflammatory HER2-positive breast cancer (BEVERLY-2): an open-label, single-arm phase 2 study. Lancet Oncol. 2012;13:375-84.

55. Hurvitz SA, Andre F, Jiang Z, Shao Z, Mano MS, Neciosup SP, et al. Combination of everolimus with trastuzumab plus paclitaxel as first-line treatment for patients with HER2-positive advanced breast cancer (BOLERO-1): a phase 3, randomised, double-blind, multicentre trial. Lancet Oncol. 2015;16:816-29.

56. Peter S, Franco C, Zhi LT, Sherene L. Oncogene addiction and immunity: clinical implications of tumour infiltrating lymphocytes in breast cancers overexpressing the HER2/neu oncogene. Curr Opin Oncol. 2014;26(6):562-7.

57. Arnould L, Gelly M, Penault-Llorca F, Benoit L, Bonnetain F, Migeon C, et al. Trastuzumab-based treatment of HER2-positive breast cancer: an antibody-dependent cellular cytotoxicity mechanism? Br J Cancer. 2006;94:259-67.

58. Roberto G, Sylvie M, Francesco F, Luisa P, Mario S, Elda T, et al. Pilot study of the mechanism of action of preoperative trastuzumab in patients with primary operable breast tumors overexpressing HER2. Clin Cancer Res. 2004;10(17):5650-5.

59. Stagg J, Loi S, Divisekera U, Ngiow SF, Duret H, Yagita H, et al. Anti-ErbB-2 mAb therapy requires type I and II interferons and synergizes with anti-PD-1 or anti-CD137 mAb therapy. Proc Natl Acad Sci USA. 2011;108:7142-7.

60. Iwata TN, Ishii C, Ishida S, Ogitani Y, Wada T, Agatsuma T. A HER2-targeting antibody-drug conjugate, trastuzumab deruxtecan (DS-8201a), enhances antitumor immunity in a mouse model. Mol Cancer Ther. 2018;17:1494-503.

61. Loi S, Giobbie-Hurder A, Gombos A, Bachelot T, Hui R, Curigliano $\mathrm{G}$, et al. Pembrolizumab plus trastuzumab in trastuzumabresistant, advanced, HER2-positive breast cancer (PANACEA): a single-arm, multicentre, phase 1b-2 trial. Lancet Oncol. 2019;20:371-82

62. Hara H, Shoji H, Takahari D, Esaki T, Machida N, Nagashima K, et al. Phase I/II study of ramucirumab plus nivolumab in patients in second-line treatment for advanced gastric adenocarcinoma (NivoRam study). J Clin Oncol. 2019;37(4s:suppl). (abstr 129)

63. Koizumi W, Narahara H, Hara T, Takagane A, Akiya T, Takagi M, et al. S-1 plus cisplatin versus S-1 alone for first-line treatment of advanced gastric cancer (SPIRITS trial): a phase III trial. Lancet Oncol. 2008;9:215-21.

64. Boku N, Ryu M, Oh D, Oh S, Chung H, Lee K, et al. Nivolumab plus chemotherapy versus chemotherapy alone in patients with previously untreated advanced or recurrent gastric/ gastroesophageal junction (G/GEJ) cancer: ATTRACTION-4 (ONO-4538-37) study. Ann Oncol. 2020;31(4s):S1192.

65. Moehler M, Shitara K, Garrido M, Salman P, Shen L, Wyrwicz L, et al. Nivolumab (nivo) plus chemotherapy (chemo) versus chemo as first-line (1L) treatment for advanced gastric cancer/ gastroesophageal junction cancer (GC/GEJC)/esophageal adenocarcinoma (EAC): First results of the CheckMate 649 study. Ann Oncol. 2020;31(4s):S1191.

66. Rogers JE, Xiao L, Trail A, Blum Murphy M, Palmer M, Ajani JA. Nivolumab in combination with irinotecan and 5-fluorouracil (FOLFIRI) for refractory advanced gastroesophageal cancer. Oncology. 2020;98:289-94.

67. Janjigian Y, Chou JMS, Momtaz P, Sanchez-Vega F, Shcherba M, et al. First-line pembrolizumab (P), trastuzumab (T), capecitabine (C) and oxaliplatin $(\mathrm{O})$ in HER2-positive metastatic esophagogastric adenocarcinoma (mEGA). J Clin Oncol. 2019;37(4s:suppl) (abstr 62)

68. Wilke H, Muro K, Van Cutsem E, Oh S-C, Bodoky G, Shimada Y, et al. Ramucirumab plus paclitaxel versus placebo plus paclitaxel in patients with previously treated advanced gastric or gastro-oesophageal junction adenocarcinoma (RAINBOW): a double-blind, randomised phase 3 trial. Lancet Oncology. 2014;15:1224-35.

69. Yoshida K, Kodera Y, Kochi M, Ichikawa W, Kakeji Y, Sano T, et al. Addition of docetaxel to oral fluoropyrimidine improves efficacy in patients with stage III gastric cancer: interim analysis of JACCRO GC-07, a randomized controlled trial. J Clin Oncol. 2019; 37(15):1296-304.

70. Nukatsuka M, Nakagawa F, Saito H, Sakata M, Uchida J, Takechi T. Efficacy of combination chemotherapy using a novel oral chemotherapeutic agent, TAS-102, with irinotecan hydrochloride on human colorectal and gastric cancer xenografts. Anticancer Res. 2015;35(3):1437-45.

71. Weber J, Mandala M, Del Vecchio M, Gogas HJ, Arance AM, Cowey CL, et al. Adjuvant nivolumab versus ipilimumab in resected stage III or IV melanoma. N Engl J Med. 2017;377:1824-35.

72. Schmid P, Cortes J, Pusztai L, McArthur H, Kummel S, Bergh $\mathrm{J}$, et al. Pembrolizumab for early triple-negative breast cancer. N Engl J Med. 2020;382:810-21.

73. Yamaguchi K, Bang Y, Iwasa S, Sugimoto N, Ryu M, Sakai D, et al. Trastuzumab deruxtecan (T-DXd; DS-8201) in patients with HER2-low, advanced gastric or gastroesophageal junction (GEJ) adenocarcinoma: Results of the exploratory cohorts in the phase 2, multicenter, open-label DESTINY-Gastric01 study. Ann Oncol. 2020;31:4s (abstr 1422MO).

Publisher's Note Springer Nature remains neutral with regard to jurisdictional claims in published maps and institutional affiliations. 